\title{
4th Clinical Sports Medicine Conference - Injury Preven- tion and Protection of the Health of the Athlete, 13 - 15 October 2010, Cape Town
}

\section{Oral presentations}

\section{PREVALENCE OF MUSCULOSKELETAL PAIN AMONG THE SOUTH AFRICAN FEMALE SENIOR NATIONAL HOCKEY PLAYERS}

Ellapen TJ, Abrahams S, Desai FA, Narsigan S, Van Heerden SJ

Discipline of Sports Science (Biokinetics), Faculty of Health Science, University of KwaZulu-Natal

Background. Field hockey is a team sport in which the competing teams attempt to score into the opposing team's goal. In an attempt to score goals, players sprint (with possession of the ball) making sudden changes in the direction of movement around their opponents, which increases the risk of musculoskeletal injury and pain.

Objectives. This study documented the prevalence of hockey-related musculoskeletal pain among the South African female senior national hockey team $(N=30)$.

Subjects. All subjects were members of the South African national senior hockey team $(N=30)$.

Methods. Subjects completed a self-report questionnaire which gathered their biographical, epidemiological and exercise history over the last 12 months. The following descriptive statistics (mode, mean, frequency, percentages) and inferential statistics (chi-square set at a probability of 0.05 ) were used to analyse the data.

Results. Twenty-eight (93.33\%) of the cohort sustained musculoskeletal pain within the last 12 months $(p<0.001)$. The most prevalent anatomical sites that sustained hockey-related musculoskeletal pain were the ankles $(24.14 \%)$, lower back and hip $(22.41 \%)$, lower leg $(13.79 \%)$, knees $(12.07 \%)$ and thighs $(10.34 \%)(p<0.01)$. The predisposing mechanisms for producing the hockey-related musculoskeletal pain were rapid, rotational movements $(43.75 \%)$, collisions with players $(18.75 \%)$, and being struck with the ball $(12.5 \%)$ $(p<0.01)$.

Conclusion. The South African senior female hockey team experienced a high prevalence of hockey-related musculoskeletal pain, especially at their ankles, lower back and hip areas.

\section{PREVALENCE OF KARATE-RELATED MUSCULOSKEL- ETAL PAIN AMONG SKISA KARATEKAS}

Ellapen TJ, Abrahams S, Desai FA, Narsigan S, Van Heerden HJ

Discipline of Sports Science (Biokinetics), Faculty of Health Science, University of KwaZulu-Natal

Background. Over the past decade, there has been heightened interest in the philosophy, training habits, nutrition and epidemiology of martial arts athletes. Despite this interest in the martial arts, there is a dearth of research publications on the epidemiology and training history of unique karate martial arts athletes, more specifically Shotokhan Karate-Do-International South Africa (SKISA) karatekas.
Objectives. This study investigated the prevalence of karate-related musculoskeletal pain among SKISA athletes.

Subjects. One hundred and sixty karatekas participated in a retrospective epidemiological investigation by voluntary informed consent. Subjects were martial art practitioners belonging to SKISA who took part in their 2010 National SKISA Kanazawa-Cup Tournament.

Methods. The subjects' training history and epidemiology of karaterelated musculoskeletal pain were gathered by means of a validated musculoskeletal pain questionnaire, which was adapted for use in this study. Descriptive statistics which included the mode, mean, frequency, percentages and inferential statistics comprising of chisquare tests (with the probability set at 0.05 ) were employed in the statistical analysis.

Results. Out of the 160 karatekas who completed the questionnaire, $61.88 \%(N=99)$ experienced karate-related musculoskeletal pain $(p<0.01)$. The prevalence of karate-related musculoskeletal pain to specific anatomical sites were hand $(18.64 \%)$, feet $(16.95 \%)$, leg $(14.41 \%)$, knee $(12.29 \%)$, back $(11.86 \%)$, shoulder $(8.47 \%)$, neck $(4.24 \%)$, elbow $(2.12 \%)$, and other $(11 \%)(p<0.01)$. The predisposing mechanism producing the karate-related musculoskeletal pain reported was direct physical trauma $(59.59 \%)$ from semi-contact $(p<0.01)$. The other factors contributing to karate-related musculoskeletal pain were overuse injuries, non-compliance to rehabilitation programmes and not seeking medical attention when musculoskeletal pain was initially experienced.

Conclusion. SKISA athletes experienced a high prevalence of karaterelated musculoskeletal hand and foot pain.

\section{A COMPARISON OF PERCEIVED VERSUS ACTUAL CARDIOVASCULAR DISEASE RISK IN BLACK URBAN MALES AND FEMALES}

\section{Jackson LM, Christie CJ}

Department of Human Kinetics and Ergonomics, Rhodes University, Grahamstown

Background. Cardiovascular disease (CVD) is becoming increasingly prominent in developing countries, such as South Africa, and particularly in previously 'risk-free' population groups, such as black South Africans. However, aligned with this is the belief that many black men and women are CVD risk-free.

Objectives. The aim of this research was to compare perceived versus actual CVD risk in black males and females from an urban working population in the Eastern Cape.

Subjects. Black urban males and females with a mean age of 41.6 years took part in the study $(N=291)$.

Methods. Obesity, hypertension, type 2 diabetes, and hypercholesterolaemia were considered as CVD risks. Body mass index (BMI) was calculated from measures of stature and mass. Obesity was classified according to BMI classification categories. The blood pressure of participants was assessed with a mercury sphygmomanom- 
eter, readings from which were then classified according to JNC 7 hypertension guidelines. Total blood glucose and cholesterol were assessed using an Accutrend GC/GCT reflectance photometer and classified according to WHO guidelines for type 2 diabetes, and NCEP guidelines for the classification of hypercholesterolaemia. An Adult Health Questionnaire (AHQ) contained questions on individuals' perceptions of the aforementioned risk factors. Participants were required to answer if they perceived themselves to be at risk. These perceived responses were then compared with objective measures.

Results. Although $93.2 \%$ of the female sample was classified as overweight/obese, only $44.6 \%$ perceived themselves to be overweight. Within the male sample, $18.2 \%$ of individuals perceived themselves as overweight, while $44.8 \%$ were classified as such Thirty-five per cent of the females perceived that they were hypertensive, which was confirmed in $36 \%$ of the sample. In comparison, only $17 \%$ of males perceived themselves to be hypertensive, while $32 \%$ were hypertensive. While $9 \%$ of the females believed they had hypercholesterolaemia, $16 \%$ fulfilled the criteria for hypercholesterolaemia. With the males, $11 \%$ were hypercholesterolaemic, compared with $3 \%$ who believed they were. While $10 \%$ and $2 \%$ of males and females perceived themselves to have type 2 diabetes, this was the case in only $2 \%$ of females and none in the males.

Conclusions. There is inconsistency between perceived and actual CVD risk with a tendency for individuals to underestimate their level of risk.

\section{THE IMPACT OF THE FREQUENCY AND DURATION OF EXERCISE PROGRAMMES ON THE FITNESS LEVELS OF 11 - 13-YEAR-OLD CHILDREN}

\section{Narsigan S}

Discipline of Sports Science (Adapted Sport Science), Faculty of Health Science, University of KwaZulu-Natal

Background. Changes to the variables of exercise programmes greatly affect the outcome. Investigations have been undertaken that examine the manipulation of frequency and duration on the exercise programmes of adults, but limited studies have been conducted on those of children.

Objectives. This study investigated the impact of frequency and duration of exercise programmes on the health-related fitness of 11 . 13-year-old children.

Subjects. The sample population consisted of 40 children from an urbanised area and a peri-urban area $(N=20)$. The inclusion criteria for eligibility to participate in the study were participation in exercise and sport programmes.

Methods. A quasi-experimental quantitative study (an ex post facto) compared the fitness levels between the groups. An exclusion criterion was a chronic illness that prevented them from participating in physical activities. Data were collected measuring the following dependent variables: body mass, stature, skinfold, resting heart rate, 1-minute sit-ups (abdominal strength and endurance) and 1-minute push-ups (upper body strength and endurance), 40-meter sprint (anaerobic capacity), 12-minute run (aerobic capacity), and modified sit and reach (flexibility). Non-parametric statistics comprising of mode, mean, frequency, percentages and parametric statistics (independent t-test) were employed in the statistical analysis.

Results. The evidence indicated that the children from the urban area

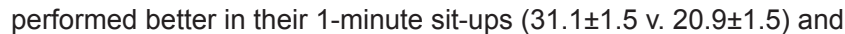

push-ups $(22.4 \pm 1.7$ v. $13.4 \pm 1.6)$, 40 -meter dash $(6.8 \pm 0.9$ v. $7.8 \pm 0.1)$ and 12 -minute run $(1920 \mathrm{~m} \pm 97.3$ v. $1682 \mathrm{~m} \pm 87.9)(p<0.05)$. In addition, children who resided in the urban area had a low per cent body fat compared with their peri-urban counterparts.

Conclusion. Data showed that children from the urban area had superior health-related fitness levels compared with those from the peri-urban area.

\section{A COMPARISON OF INTERMITTENT AND CONTINU- OUS CYCLING TRAINING ON THE CARDIOVASCULAR HEALTH OF POSTMENOPAUSAL WOMEN}

\section{Sheppard BJ, Christie CJ}

Department of Human Kinetics and Ergonomics, Rhodes University, Grahamstown

Background. The risk of cardiovascular disease increases exponentially in postmenopausal women. Exercise as a treatment has not been fully elucidated, particularly aerobic exercise training of an intermittent nature.

Objectives. To compare the effect of two types of aerobic cycling exercise (intermittent versus continuous) on selected health measures in this population group.

Subjects. Eighteen previously sedentary, postmenopausal women (defined as no menses for 12 consecutive months) between 50 and 75 years old and in good health (non-smokers, no hypertension, diabetes mellitus, and not taking any form of hormone replacement therapy) were recruited from the Grahamstown area in the Eastern Cape province of South Africa.

Methods. Subjects were randomly divided into two groups of equal numbers, representing the intermittent (EXP) and the continuous (CON) protocol, both involving stationary cycling of a progressive nature (for 8 weeks). All subjects attended three supervised exercise sessions per week, with an initial duration of 20 minutes per session, increasing to 28 minutes per session in the final 4 weeks. The training load for both groups was matched to allow any benefits to be directly attributed to the specific protocol. Selected measurements (body mass, stature, waist and hip girth, blood pressure, reference heart rate and a full blood lipid profile) were obtained at weeks 0,4 and 8 respectively. For the duration of the intervention, subjects were requested to maintain their habitual dietary intake and not to embark on any weight loss programme.

Results. There were no significant $(p<0.05)$ alterations in body mass, waist and hip circumference, blood pressure, high density lipoprotein and triglyceride concentrations at the end of the 8-week period in either group. However, significant $(p<0.05)$ reductions were observed in total cholesterol and low density lipoprotein concentrations after the initial 4 weeks within the EXP group only. Thereafter these measures increased significantly to pre-measurement values at 8 weeks.

Conclusions. Although 4 weeks of intermittent cycle training resulted in an improvement in blood lipid concentrations, these benefits were no longer apparent at the end of the 8 weeks. It can therefore be concluded that 8 weeks of cycling training, whether of an intermittent or continuous nature, resulted in no positive alterations of certain cardiovascular risk measures in a cohort of postmenopausal women. 
COMPARATIVE ULTRASOUND STUDY OF ACUTE LATERAL ANKLE LIGAMENT INJURIES REHABILITATED WITH STANDARD AND JUMP STRETCH FLEX BAND PROGRAMMES

\section{Van der Linde E, Oschman Z}

Section Sports Medicine, Faculty of Health Sciences, University of Pretoria

Background. Lateral ankle ligament injuries are very common in sport. We were interested in finding a suitable rehabilitation method to get sportsmen and women back to the playing field as soon as possible with minimal risk.

Objectives. To establish whether rehabilitation with the jump stretch flex band (JSFB) programme would be superior to standard ankle rehabilitation of acute lateral ankle ligament injuries.

Subjects. All rugby and non-rugby players with acute lateral ankle ligament injuries were considered potential subjects.

Methods. Ultrasound examinations were done 72 hours post injury and repeated 2 weeks post rehabilitation. Swelling, thickness, appearance of ligaments and recovery time were compared in grades 1 and 2 ankle ligament injuries. After we had distinguished the amount of tissue damage, we started a rehabilitation programme where the patients were divided into group A (study group) or group B (control group). Group A received JSFB rehabilitation from day 4 post injury onwards, whereas group $B$ started with a standard ankle rehabilitation programme from day 10 post injury onwards. An ultrasound examination was done on each patient every 2 weeks to compare the amount of tissue healing in both groups. Only well-researched and acknowledged techniques were used for the study.

Results. The anterior talo-fibular ligament (ATFL) of both groups A and $B$ tended to increase in size with the JSFB over 2-week time intervals $(p=0.0025, \mathrm{U}=0$ for grade $1 ; p=0.0038, \mathrm{U}=0$ for grade 2). The fluid in the anterior recess decreased more with the JSFB $(p=0.0426, \mathrm{U}=5)$ for Ggade 1 injuries and less than the standard ankle rehabilitation programme for grade 2 injuries $(p=0.33, \mathrm{U}=12.0)$. Fluid from the posterior recess was decreased more for both grade 1 and 2 injuries with the JSFB $(p=0.1184, \mathrm{U}=30.5$ for grade $1 ; p=0.47$, $\mathrm{U}=22.0$ for grade 2 ). The recovery time (RT) of grade 1 ankle injuries rehabilitated with a JSFB were on average 11 days shorter than grade 1 ankle injuries rehabilitated on a standard ankle rehabilitation programme $(p=0.0026, U=0)$. Similar results were found with grade 2 ankle injuries. The recovery times (RT) of grade 2 ankle injuries on the JSFB were on average 12 days shorter than those of grade 2 ankle injuries rehabilitated on a standard ankle rehabilitation programme $(p=0.0081, \mathrm{U}=1.5)$. We only had one patient with a re-injury 2 months post rehabilitation on an ankle rehabilitated with the standard ankle rehabilitation programme, and no re-injuries for ankles rehabilitated on the JSFB. In general, there was a tendency for the appearance of the ATFL to have a more significant change from abnormal to normal with the JSFB than that of a standard ankle rehabilitation programme for grades 1 and 2 ankle ligament injuries ( $p=0.0699$ for grade $1 ; p=0.0606$ for grade 2 ). Thus the JSFB proved to be more effective.

Conclusions. Rehabilitation with the JSFB programme proved to be superior to standard ankle rehabilitation. Grades 1 and 2 ankle ligament injuries recovered sooner, resulting in stronger ligaments, and no re-injuries were reported. The general public may benefit in returning to their daily activities much sooner, and athletes may resume normal competition without any delay.

\section{RANGE OF MOTION DIVERGES WITH INCREASING AGE FOR THE COL5A1 BSTUI GENOTYPES}

\section{Brown JC, Miller C-J, Schwellnus MP, Collins M}

UCT/MRC Research Unit for Exercise Science and Sports Medicine, Dept of Human Biology, Faculty of Health Sciences University of Cape Town

Background. Both an increased and decreased flexibility/joint range of motion (ROM) is a modifiable risk factor for musculoskeletal softtissue injuries. Mutations within the COL5A1 gene have been shown to cause heritable disorders of connective tissue, which have a unifying symptom of joint hypermobility. Furthermore, a COL5A1 gene sequence variant, the BstUI restriction fragment length polymorphism (RFLP), is associated with range of motion (ROM) measurements in a population of individuals with a history of Achilles tendon injuries.

Objectives. To investigate the possible association between the COL5A1 gene BstUI RFLP and sit and reach ROM in an apparently healthy and physically active population.

Subjects. Three hundred and twenty-five (of which 204 were males) white, apparently healthy and physically active subjects were recruited from local fitness centres and running events.

Methods. The sit and reach (SR) test and genotyping of the BstUI RFLP $(\mathrm{C} / \mathrm{T})$ within the 3'-untranslated region of the COL5A1 gene was performed on all subjects. Detailed medical questionnaires on training status, injury and medical history were also completed by all subjects.

Results. While the COL5A1 BstUI RFLP genotypes were not associated with SR ROM in the 'young' ( $<35$ years) subjects $(N=197$, $p=0.626$ ), there was a significant difference in the mean SR ROM between the genotype groups of the 'old' ( $\geq 35$ years) subjects (TT=225 $\pm 96 \mathrm{~mm}, \quad T C=245 \pm 100 \mathrm{~mm}, \quad C C=321 \pm 108 \mathrm{~mm}, \quad N=96$, $p=0.017)$. A significant interaction between age and COL5A1 BstUI genotype explained the difference in SR mean scores with increasing age. Furthermore, only two factors, gender and BstUl genotype, explained a significant proportion $(22.8 \%)$ of the variance in SR ROM in the 'old' group.

Conclusions. The COL5A1 BstUI RFLP is associated with SR ROM, particularly with increasing age, and this finding partially explains the results of previous reports from this laboratory. This study suggests that genotype, along with other common intrinsic and extrinsic phenotypic factors, should be considered when assessing ROM.

\section{IMPORTANCE OF TACHOGRAM LENGTH AND PERIOD OF RECORDING DURING THE NON-INVASIVE INVESTI- GATION OF THE AUTONOMIC NERVOUS SYSTEM}

Grant $C^{1}{ }^{1}$, Janse van Rensburg DC ${ }^{1}$, Strydom $\mathbf{N}^{2}$, Viljoen $\mathbf{M}^{3}$

${ }^{1}$ Section Sports Medicine, Faculty of Health Sciences, University of Pretoria

${ }^{2}$ Department of Statistics, University of Pretoria

${ }^{3}$ Department of Physiology, Faculty of Health Sciences, University of Pretoria

Background. Various disciplines, including research in health and prevention in sports medicine, use quantification of heart rate variability as an indicator of autonomic function without recognising the importance of using standardised methodologies. 
Objective. The aim of this study was to assess the influence of tachogram length and period of recording on heart rate variability (HRV) indicator values.

Methods. To test the influence of the length of the recording time, HRV indicator values calculated from 180, 300, 420, and $600 \mathrm{sec}-$ onds recording periods (supine and standing) were compared. Also, individual 3-minute periods (0 - $180 \mathrm{~s}, 180$ - $360 \mathrm{~s}, 360$ - $540 \mathrm{~s}$ ) from 10-minute (supine and standing) tachograms were compared.

Results. In the supine position, vagal efferent HRV indicators were not influenced by tachogram length varying between 3 and 10 minutes $(p>0.05)$. Supine HRV indicator values representing combined sympathetic and parasympathetic influences were tachogram length-dependent $(p<0.05)$. During orthostatic stress, all HRV indicators were tachogram length-dependent $(p<0.05)$. Upon standing up, marked vagal withdrawal and sympathetic activation occurred. Vagal withdrawal tapered off during the 3 - 6-minute period after rising, while indicators representing combined vagal and sympathetic activation were totally reversed.

Conclusions. During the application of orthostatic stress the exact starting point of recording, as well as the length of recording, is critical due to the activation and normalisation of homeostatic mechanisms. Starting the tachogram recording too late will miss out part of the initial response to change in body position. Longer recording times will give a combination of values recorded during the stress response and values obtained after stabilisation in the standing position.

\section{IMPACT OF CLOTHING AND PROTECTIVE GEAR ON PHYSIOLOGICAL, PERCEPTUAL AND PERFORMANCE RESPONSES DURING SIMULATED RUGBY ACTIVITY}

\section{Cannon MJ, Christie CJ}

Human Kinetics and Ergonomics Department, Rhodes University, Grahamstown

Background. There is a lack of information regarding the influence of clothing and protective gear on rugby players' physiological, perceptual and performance responses during simulated rugby activity.

Objectives. To compare players' responses when wearing minimal rugby clothing and no protective gear (control condition - CC), and when wearing full rugby clothing and protective gear (experimental condition - EC).

Subjects. Eight Makulu Blue rugby players from the Rhodes University internal rugby league were selected as subjects.

Methods. Players performed a simulated 40-minute $(2 \times 20$-minute $)$ intermittent running rugby protocol which excluded contact rugby activities. Players performed the protocol between two points, set 20 meters apart, by standing, walking, jogging, striding and sprinting intermittently when instructed to do so by the tester. This protocol was very stop/start and intermittent in nature, which matched the demands of a real rugby match. Test subjects performed this protocol wearing either the control kit or the experimental kit. With both protocols, physiological (skin temperature, fluid loss and heart rate) and perceptual measures (ratings of perceived exertion and thermal discomfort) were recorded prior to testing, and then at 10-minute intervals during the protocol. Prior to testing and following both conditions, players performed a multi-stage 20-metre shuttle run (performance measure) wearing only the control kit.
Results. The intermittent rugby protocol was the greatest stressor, with protective gear exacerbating these effects. Skin temperature increased significantly from the start to the end (0 - 40 minutes) of the EC (mean of $34.8^{\circ} \mathrm{C}$ ), while skin temperature reached a 'steady state' during the $\mathrm{CC}$ (mean of $31.9^{\circ} \mathrm{C}$ ). Heart rate increased significantly in the transition from rest to exercise and thereafter stabilised in both conditions. This excluded the last time interval when heart rate increased significantly $(p<0.01)$ in both conditions, although heart rates at this time interval were significantly higher in the EC (mean of 184 bt. $\mathrm{min}^{-1}$ ) compared with the CC (mean of 167 bt.min ${ }^{-1}$ ). During the EC, fluid loss was significantly greater $(0.79$ liter compared with 0.57 liter in the EC and CC respectively) because of an increased absolute sweat rate. Despite the increased physiological strain, players did not perceive this extra strain until the end of the EC protocol. Furthermore, players perceived significantly more thermal strain during the EC. The most important finding was that performance was significantly compromised following the EC.

Conclusion. It is contended that the intermittent nature of rugby imposes physical stress on players. The stress is greater when these players wear full rugby clothing and protective gear. The combined impact of intermittent exercise plus clothing and protective gear had a detrimental effect on performance.

\section{A PATHWAY-BASED APPROACH INVESTIGATING THE INFLAMMATORY PATHWAY AND PREDISPOSITION TO THE DEVELOPMENT OF ACHILLES TENDINOPATHY}

\section{Nell E-M, Collins M, Schwellnus MP, September AV}

UCT/MRC Research Unit for Exercise Science and Sports Medicine, Department of Human Biology, University of Cape Town

Background. Achilles tendinopathy (AT) is a multifactorial condition for which several genetic risk factors have been identified. AT is characterised by altered expression of several cytokines and inflammatory mediators. Recently, the $I L-1 \beta, I L-1 R N$ and $I L-6$ genes were found to modulate risk of AT in combination with the COL5A1 BstUI RFLP. Therefore genes encoding mediators of the $I L-1 \beta$ matrix degradation pathway (COX-2, PTGER4) and mediators of the IL-6 apoptosis pathway (CASP8, NOS3) were explored for associations to AT.

Objectives. The aim of this study was to determine whether functional polymorphisms within the COX-2, PTGER4, CASP8 and NOS3 genes, encoding proteins involved in extracellular matrix (ECM) degradation and tenocyte apoptosis, may contribute towards a risk of AT in South African (SA) and Australian (AUS) individuals.

Subjects. A total of 163 asymptomatic controls (SA CON) and 93 participants with AT (SA TEN) from SA and 210 AUS CON and 85 AUS TEN participants were recruited.

Methods. A pathway-based case-control genetic association study was conducted. Participants were genotyped for rs20417 (COX2$765 \mathrm{G}>\mathrm{C}$ ), rs4495224 (PTGER4 gene desert $\mathrm{A}>\mathrm{C}$ ), rs3834129 (CASP8-652 6Ndel) and rs1799983 (NOS3Glu298Asp).

Results. The CASP8-652 del/del genotype was significantly overrepresented in AUS TEN compared to AUS CON $(p=0.027 ; \mathrm{OR}=2.0$, $95 \% \mathrm{Cl} 1.1-3.6)$. The CASP8-652 ins/del genotype ( $p=0.029$; $\mathrm{OR}=1.8,95 \% \mathrm{Cl} 1.1-3.1)$ and PTGER4 CC genotype ( $p=0.010$; $\mathrm{OR}=4.4,95 \% \mathrm{Cl} 1.3-15)$ were significantly over-represented in AUS CON compared to AUS TEN. No associations were observed for COX2-765G>C and NOS3Glu298Asp in AUS. No genotype or allele associations were observed in SA. 
Conclusions. Variations within genes involved in the inflammatory pathway modulate risk of AT. This research emphasises that a pathway-based genetic association study may be a more effective approach to capture and understand the genetic risk factors underlying multifactorial conditions, such as AT.

\section{Poster presentations}

\section{ELECTROMYOGRAPHIC (EMG) ACTIVITY OF VASTUS MEDIALIS OBLIQUUS (VMO) AND VASTUS LATERALIS (VL) DURING ISOKINETIC KNEE EXTENSION AT VARI- OUS VELOCITIES}

\section{Neveling N, Moshoeu L}

Biokinetics, Department of Sport, Rehabilitation \& Dental Sciences, Tshwane University of Technology

Background. It has been stated that VMO:VL strength ratio should be $1: 1$ in order to maintain appropriate patella alignment, and that the response time for VMO should be less than VL. Several authors have indicated that slower response times or weaker ratios may lead to excessive lateral patella tracking, predisposing the individual to injury. Current literature shows conflicting results in this regard, with results varying from a response time favouring $\mathrm{VMO}, \mathrm{VL}$, or neither of the muscles. It has been proposed that these confounding results regarding VMO and VL electrical activity could be directly related to differences in methods, subject selection, electrode placements and level of loading.

Objectives. The purpose of the current study was to investigate whether there is a difference in EMG activity of $\mathrm{VMO}$ and $\mathrm{VL}$ during isokinetic knee extension at $60 \%$ s, $90 \%$ and $180 \%$ respectively.

Subjects. Twenty male participants who were accustomed to strength training took part in the study.

Methods. Each subject underwent three familiarisation trails with isokinetic and EMG testing prior to the study. Following a standardised warm-up on a cycle ergometer, each participant performed 5 maximal isokinetic repetitions at $60 \%, 90 \%$ s and $180 \%$ s with 1 minute rest between sets. The sequence of testing was randomised. EMG data was recorded from the VMO and VL for each repetition, with the best values being analysed.

Results. The VMO: VL ratio was $113.9 \%, 105.4 \%$, and $107.5 \%$ for $60 \%$ s, $90 \%$ and $180 \%$ respectively. No significant differences were observed between testing velocities. No significant differences in the peak EMG activity for VMO and VL were observed during isokinetic knee extension at 3 velocities.

Conclusions. This indicates that the VMO and VL contract at approximately the same intensity, with ratios being close to 1 . As the testing was not repeated on individuals, further research should investigate whether re-testing the participants would result in changes regarding EMG activity of $\mathrm{VMO}$ and $\mathrm{VL}$ at various velocities.
THE EFFECT OF NEURO-LYMPHATIC REFLEX MUSCLE ACTIVATION TECHNIQUES ON QUADRICEPS STRENGTH DURING ISOKINETIC TRAINING

\section{Neveling N, Bekker S, Tshabalala NF}

Biokinetics, Department of Sport, Rehabilitation \& Dental Sciences, Tshwane University of Technology

Background. Muscle activation techniques rely on a system of neuro-lymphatic reflexes in the body to increase the neural drive to a specified muscle. The general technique entails rubbing an activation point over a reflex area for $10-20$ seconds to increase the brain's 'awareness' of the muscle to improve performance. This technique has gained popularity in recent years, with very little scientific backing.

Objectives. To determine the effect of neuro-lymphatic reflex muscle activation techniques on quadriceps strength during isokinetic training.

Subjects. Fifteen sedentary female subjects were used in the study.

Methods. All subjects underwent three separate familiarisation sessions on the isokinetic equipment and with the general techniques of neuro-lymphatic reflex muscle activation prior to the initial testing to minimise possible training effects. A randomised, placebo-controlled design was used during the study. On day 1 of the testing subjects underwent activation on the reflex area for the quadriceps (experimental), a placebo site (placebo), or no activation at all (baseline). Isokinetic knee flexion/extension testing was performed immediately following the activation at $60 \%$ s. The testing was repeated 1 week later with alternate activation. The differences in peak torque for each testing session were compared with the baseline values using a $t$-test. Results were also analysed for a learning effect using an ANOVA.

Results. No learning effect was observed. It was further noted that no significant differences $(p>0.05)$ were observed for peak isokinetic torque for the quadriceps $(p=0.06)$, hamstrings $(p=0.17)$, or the quadriceps: hamstring ratios $(p=0.89)$.

Conclusions. Based on these values, it would appear that, at least for the quadriceps, neuro-lymphatic reflex muscle activation does not lead to an increase in muscle strength. It is plausible that the anecdotal improvements that have been commonly referred to may be linked to psychosomatic improvements through suggestion. It should be noted that the study population in this trial was small.

\section{DIFFERENCES IN MUSCLE ACTIVATION PATTERNS ACROSS THE MENSTRUAL CYCLE}

\section{Sauer JW, Neveling N}

Biokinetics, Department of Sport, Rehabilitation \& Dental Sciences, Tshwane University of Technology

Background. Research has shown that females experience more anterior cruciate ligament $(A C L)$ injuries than males. Even though anatomical and neuromuscular factors contribute to the above, it has also been suggested that this could be linked to changes in hormone levels. Oestrogen has been linked to decreased strength and increased ligament laxity in females.

Objectives. To determine whether there is a difference between the muscle strength and activation patterns in females across the menstrual cycle. 
Subjects. Seven female and eight male subjects took part in the study.

Methods. The ovulatory (corrected day 14) and follicular (corrected day 21) was estimated for each female participant from a menstrual history recall questionnaire. Each female subject was tested on each of these days, with male subjects being tested as controls on the same days. The order of the testing (ovulatory v. follicular) was randomised. For each testing session, isokinetic peak torque was assessed on an isokinetic dynamometer at $60 \%$ and $180 \%$ respectively. EMG activation patterns for rectus femoris $(R F)$, vastus lateralis $(\mathrm{VL})$, vastus medialis (VM), biceps femoris (BF), semitendinosus (ST), and medial gastrocnemius (G) was assessed during three drop landings from a $36 \mathrm{~cm}$ height.

Results. No significant differences were observed in the quadriceps peak torque or time to peak torque. The hamstrings showed a decrease in peak torque during the ovulatory phase, even though this was not significant. A significant difference $(p<0.05)$ was found in time to peak torque for isokinetic hamstring strength between the menstrual phases. EMG amplitude showed no significant difference during the menstrual phases during drop landing. Time to muscle activation changed significant $(p<0.05)$ for $\mathrm{VMO}, \mathrm{ST}$, and BF showing slower activation during the ovulatory phase compared to the follicular phase.

Conclusions. Delayed hamstring activation may predispose females to ACL injury during the ovulatory phase. This delayed activation may be linked to decreased reflex activation of the hamstrings, leading to increased anterior tibial translation and load on the ACL. This can possibly partly explain the increased risk for $A C L$ injury seen in females during the menstrual cycle. Possible shortcomings include that the phases of the cycle was not confirmed by blood analysis, and the small sample size.
THE COL5A1 GENE, ULTRA-MARATHON RUNNING PERFORMANCE AND SIT AND REACH RANGE OF MOTION

Brown JC, Miller C-J, Posthumus M, Schwellnus MP, Collins M

UCT/MRC Research Unit for Exercise Science and Sports Medicine, Department of Human Biology, Faculty of Health Sciences, University of Cape Town

Background. Endurance running performance and joint range of motion (ROM) are both multifactorial phenotypes. The COL5A1 gene has been separately associated with both endurance running performance and sit and reaches range of motion (SR ROM). SR ROM has been associated with running economy, a key predictor of endurance running performance.

Objectives. To investigate whether SR ROM is related to running performance in an ultra-endurance event and if this relationship would be associated with the COL5A1 genotype.

Subjects. Seventy-two white runners (52 male) were recruited from the Two Oceans $56 \mathrm{~km}$ ultra-marathon.

Methods. Subjects performed a pre-race SR test and donated a 5 $\mathrm{ml}$ blood sample. The cohort was genotyped for the (T/C) COL5A1 $B s t U I$ RFLP. Subjects completed a questionnaire on training history. Race times were collected after the event from the official event website.

Results. The TT genotype ( $341 \pm 41 \mathrm{mins}, N=21)$ was significantly associated $(p=0.048)$ with better overall ultra-marathon running performance in comparison to the $\mathrm{C}$ allele ( $\mathrm{TC}+\mathrm{CC}$ genotypes) (365 \pm 39 mins, $N=50)$. In contrast, the CC genotype $(304 \pm 94 \mathrm{~mm}$, $\mathrm{N}=18$ ) had significantly greater pre-race SR ROM than the T allele (TT + TC genotypes) $(241 \pm 95 \mathrm{~mm}, N=54)(p=0.018)$. Furthermore, age and COL5A1 genotype accounted for a significant proportion of the variance in overall race time (19\%). Furthermore, when divided into quartiles based on ROM and overall race time, the T allele were significantly over-represented in the 'fast-inflexible' group (T, 69\% v. $47 \%$ C; $p=0.044)$.

Conclusions. The COL5A1 genotype was independently associated with both pre-race SR ROM and performance in an ultra-endurance running event. However, there was no evidence of a direct association between ROM and performance, although the T allele was significantly over-represented in the 'fast-inflexible' group. 